\title{
Which Factors Affect Short-Term Urinary Continence and Erectile Function Recovery Following Robotic-Assisted Radical Prostatectomies (RARP)?-A Retrospective Cohort Study
}

\author{
Reyan Saghir ${ }^{1, *}$, Francesca Kum ${ }^{2}$, Noman Saghir ${ }^{3}$, Cecilia Bosco ${ }^{4}$, Mieke Van Hemelrijck ${ }^{4}$, Prokar Dasgupta ${ }^{5}$, Paul Cathcart ${ }^{5}$, \\ Christian Brown ${ }^{5}$, Rick Popert ${ }^{5}$, Kathie Wong ${ }^{2}$, Lorenzo Marconi ${ }^{6}$, and Ben Challacombe ${ }^{5}$
}

\author{
${ }^{1}$ Foundation Year Doctor, GKT School of Medicine, King's College London, Hodgkin Building, Newcomen St, London SE1 1UL, United Kingdom \\ ${ }^{2}$ Urology Registrar, Guy's Hospital Urology Department, Great Maze Pond, London SE1 9RT, United Kingdom \\ ${ }^{3}$ Plastics Core Trainee, Pinderfields General Hospital, Aberford Road, Wakefield, WF1 4DG, United Kingdom \\ ${ }^{4}$ Division of Cancer Studies, Cancer Epidemiology Group, King's College London, London, United Kingdom \\ ${ }^{5}$ Consultant Urological Surgeon, Guy's Hospital Urology Department, Great Maze Pond, London SE1 9RT, United Kingdom \\ ${ }^{6}$ Robotic Fellow, Guy's Hospital Urology Department, Great Maze Pond, London SE1 9RT, United Kingdom
}

*Corresponding author: Reyan Saghir, Foundation Year Doctor, GKT School of Medicine, King's College London, Hodgkin Building, Newcomen St, London SE1 1UL, United Kingdom, E-mail: reyansaghir@gmail.com

Received: 03 May, 2021 | Accepted: 05 May, 2021 | Published: 10 May, 2021

Citation: Saghir R, Kum F, Saghir N, Bosco C, Van Hemelrijck M, et al. (2021) Which Factors Affect Short-Term Urinary Continence and Erectile Function Recovery Following Robotic-Assisted Radical Prostatectomies (RARP)?-A Retrospective Cohort Study. J Surg Open Access 7(3): dx.doi. org/10.16966/2470-0991.238

Copyright: (c) 2021 Saghir R, et al. This is an open-access article distributed under the terms of the Creative Commons Attribution License, which permits unrestricted use, distribution, and reproduction in any medium, provided the original author and source are credited.

\begin{abstract}
Introduction: To evaluate peri-operative variables in men undergoing Robotic-Assisted Radical Prostatectomy (RARP) and their relationship with short-term outcomes at a tertiary referral centre. These variables can inform surgical planning and intra-operative management to improve patient outcomes.

Patients and Methods: A retrospective analysis of consecutive 331 RARPs over a 16-month period performed by five surgeons was conducted. Primary endpoints included undetectable PSA at 6 weeks, urinary continence defined as 0-1 pad use for security leakage at 6 months, and erectile function defined as ability to have intercourse with or without PDE-5 inhibitors, pump or intercavernosal injections at 6 months. Models for multiple logistic regression analysis were fitted for prediction of each of the three endpoints.

Results: Using multiple logistic regression analysis increased postoperative PSA levels were found significantly associated to; increased preoperative PSA levels (Odds Ratio $[\mathrm{OR}]=1.05,95 \%$ Confidence Interval $[\mathrm{Cl}]=1.01-1.09, \mathrm{p}=0.01$ ), presence of positive surgical margins less than $3 \mathrm{~mm}$ compared to negative margins ( $\mathrm{OR}=4.7,95 \% \mathrm{Cl}=1.87-11.81, \mathrm{p}=0.00)$ and presence of metastatic lymph nodes vs. benign $(\mathrm{OR}=4.74,95 \% \mathrm{Cl}=0.96-23.99, \mathrm{p}=0.05)$, whilst unilateral and bilateral nerve sparing compared to non-nerve sparing were significantly associated to undetectable levels of PSA postoperatively respectively ( $\mathrm{OR}=0.37$ and $0.3,95 \% \mathrm{Cl}=0.15-0.91$ and $0.12-0.71, \mathrm{p}=0.03$ and 0.01 ). Continence outcomes were found to be significantly associated with increasing age $(\mathrm{OR}=1.04,95 \% \mathrm{Cl}=1.01-1.08, \mathrm{p}=0.02)$ and differing amongst the operating consultants $(\mathrm{OR}=0.33,95 \% \mathrm{Cl}=0.13-0.82, \mathrm{p}=0.02)$, whilst erectile dysfunction was also found to be significantly associated with increasing age $(\mathrm{OR}=1.78, \mathrm{Cl}=1.49-2.28, \mathrm{p}=0.00)$.

Conclusion: The level of nerve sparing performed needs to be considered in relation to the presence of positive surgical margins remaining as this can significantly impact postoperative PSA levels in the short-term and reduce the chances of a biochemical cure. The interoperator variability amongst operating consultants in relation to continence outcomes proves interesting and needs further investigation.
\end{abstract}

Keywords: Robotic assisted radical prostatectomy; PSA; Continence; Erectile dysfunction; RARP; Prostate cancer

\section{Introduction}

Robotic-Assisted Radical Prostatectomy (RARP) is currently a gold standard treatment for localised prostate cancer [1]. Variations in prostatectomy technique include nerve-sparing, limited or extended Pelvic Lymph Node Dissection (PLND). During nerve-sparing, the closely associated neurovascular bundles are preserved to allow for spontaneous penile erections to be maintained postoperatively. A limited PLND refers to only the lymph nodes surrounding and in close association to the prostate found in the external iliac and obturator fossa areas, meanwhile extended PLND remove nodes further away including hypogastric, pre-sacral and pre-sciatic regions [2].

Development of the DaVinci (Intuitive ${ }^{\star}$ ) surgical system and preliminary trialling of its application in a urological setting led to the first documented RARP occurring in early $2000[1,3,4]$. Following this, 
use of the DaVinci system has been adopted in many centres worldwide. Guy's and St Thomas' Hospitals was one of the first to pioneer the use of robotic assistance in the urology setting in the United Kingdom in 2004, which is now practiced across the globe. Comparative to Laparoscopic Radical Prostatectomy (LRP), RARP is highly effective with increased precision and accuracy, leading to improved patient outcomes. The odds of biochemical recurrence, urinary incontinence and Erectile Dysfunction (ED) are lower following RARP compared to LRP, as found in systematic reviews by Ficarra V, et al. and Montorsi F, et al. [5,6]. Although outcomes have improved since the introduction and use of RARP, postoperative Prostate Specific Antigen (PSA) levels, continence and erectile function are still concerning to patient's disease-free survival and quality of life.

This study aims to evaluate perioperative variables in men undergoing RARP, to establish any significant correlations affecting these short-term postoperative outcomes of PSA levels, continence and ED. By doing so we hope to develop our understanding and aid patient management and postoperative care to ensure optimal patient recovery is achievable.

\section{Patients and Methods}

This was a retrospective analysis of all RARPs performed at a high volume tertiary robotic centre between January 2016 and April 2017. A total of 382 RARPs were performed of which 331 were statistically analysed. 51 cases were excluded from analysis due to insufficient postoperative data or follow-up was not received at our trust.

Each patient received a standard preoperative workup: PSA, MRI and appropriate prostate biopsies were taken within 14 days of first referral. The Briganti nomogram forms the basis for lymphadenectomy with a 5\% threshold for lymph node involvement alongside PSMAPET code scanning results. All patients were discussed at the MultiDisciplinary Team (MDT) meeting in which tumour grade, stage and lymph node dissection was decided, with a management plan agreed and as per the network target by day 62 from initial presentation to the first definitive treatment [7].

All operative procedures were carried out according to local trust protocol. Patients received prophylactic gentamycin and intravenous co-amoxiclav at induction. Patients were positioned in the Reverse Trendelenburg position with end docking and standard 6 port access for the dual console Da Vinci Xi (Intuitive ${ }^{\oplus}$ ) Surgical System. Postoperatively, all patients were mobilized on the $1^{\text {st }}$ postoperative day with their urethral catheter in situ and discharged with low molecular weight heparin injections for 28 days. Patients then had a nurse-led trial without catheter at week 1 and surgeon review at 6 weeks postRARP.

Data was collected on 15 perioperative variables and was correlated to three-short term postoperative outcomes: PSA levels at 6 weeks, urinary continence and ED at 6 months. An undetectable PSA was deemed as $<0.03 \mathrm{ng} / \mathrm{L}$. Continence endpoints were defined by individuals either fully continent or using a security pad for light leakage followed by increased number of pad usage per day, at 6 months postoperatively, and the endpoint defining complete ED was ED refractory to oral agent, pump or injections. The perioperative variables examined included: patient demographics; operating consultant; preoperative PSA, Gleason score, amount of nerve sparing (none, unilateral or bilateral); tumour staging; distance of surgical margins; presence of seminal vesicle invasion and grading of nodes removed.

Data collection and analysis was performed using RStudio (Version 1.1.456). Multiple logistic regression analyses were performed to identify predictors for each of the three endpoints. In order to increase power to the test, endpoints for the analysis were defined as

- $\quad$ Post-operative PSA $0 \leq 0.03 \mathrm{ng} / \mathrm{L}, 1 \geq 0.03 \mathrm{ng} / \mathrm{L}$

- Continence $0=$ Fully Continent $1=$ any incontinence

- Post-operative erectile Function $0=$ no ED $1=$ any ED

For descriptive purposes, endpoints with subcategories are also presented in graphs and tables. If a variable did not have enough number of events for the outcomes of interest, then it was excluded from the analysis.

This study has been registered as an audit at our institution (Ref No: 8077) and all work has been reported in line with the STROCSS criteria [8]. No ethical approval or funding was required for this study.

\section{Results}

A total of 382 RARPs were carried out between January 2016 to April 2017 (mean 24/month), of which 331 underwent full analysis. 51 cases were excluded due to insufficient postoperative data or follow-up was not received at our trust. Cases were performed by five experienced Urologists at a single institution.

Mean patient age was 62.22 \pm 7.45years (IQR) (range: 38-78years). Mean preoperative PSA was $10.12 \pm 7.86$ (SD) (range: 1.20-68.00) $\mathrm{ng} / \mathrm{ml}$, and using the new Epstein Gleason scoring system the modal grade group was 2 indicative of a Gleason score 3+4=7 [9].

152/331 (45.9\%) patients underwent a bilateral nerve sparing procedure, unilateral in $109(32.9 \%)$ and no nerve sparing in 70 (21.1\%). Histopathological analysis of the prostate specimen removed from patients showed that $21(6.3 \%)$ had invasion of the seminal vesicles.

Of 71 patients whom had a lymphadenectomy, 63 were benign (88.7\%), 3 (4.22\%) had micrometasatsis and $5(7.04 \%)$ were metastatic.

Documented complications were minimal, 6/331 (1.81\%), and Clavien-Dindo I/II only.

Tumour and patients' characteristics can be found in table 1.

\section{Associations between perioperative and short-term postoperative outcomes}

PSA level at 6 weeks: At 6 weeks post RARP, a routine PSA level was performed. $278(84.0 \%)$ patients had an undetectable PSA at 6 weeks (PSA $<0.03 \mathrm{ng} / \mathrm{L}$ ), thus indicating biochemical cure. Twentyeight patients $(8.46 \%)$ had PSA 0.03 to $<0.1$ and the remainder 25 (7.55\%) had PSA 0.1 to $<0.6$.

On analysis we found increased preoperative PSA level to be significantly associated to increased postoperative PSA levels at 6 weeks $(\mathrm{OR}=1.05, \mathrm{p}=0.01)$. We also found positive surgical margins less than or equal to $3 \mathrm{~mm}$ in length associated with increased postoperative PSA levels at 6 weeks $(\mathrm{OR}=4.70, \mathrm{p}=0.00)$ compared to negative surgical margins. Metastatic lymph nodes removed were additionally found to be significantly associated to increased postoperative PSA levels $(\mathrm{OR}=4.74, \mathrm{p}=0.05)$. Whilst unilateral and bilateral nerve sparing were identified as significantly protective factors in reducing postoperative PSA levels at 6 weeks comparative to non-nerve sparing RARP $(\mathrm{OR}=0.37,0.30$ and $\mathrm{p}=0.03,0.01$ respectively) (Table 2$)$.

Urinary continence at 6 months: At 6 months, 75.8\% (251/331) patients were either fully continent or using a security pad (Figure 1).

When performing the multiple logistic regression analysis all perioperative variables were included. The results found firstly 
Table 1: Tumour and patient characteristics.

\begin{tabular}{|c|c|c|c|c|c|c|c|c|c|}
\hline & \multicolumn{3}{|c|}{ Urinary Incontinence } & \multicolumn{3}{|c|}{ Erectile Dysfunction } & \multicolumn{3}{|c|}{ Post-Operative PSA } \\
\hline & No & Yes & Overall & No & Yes & Overall & $<0,03$ & $>=0,03$ & Overall \\
\hline & $(n=207)$ & $(n=124)$ & $(n=331)$ & $(n=31)$ & $(n=300)$ & $(n=331)$ & $(n=278)$ & $(n=53)$ & $(n=331)$ \\
\hline \multicolumn{10}{|c|}{ Age } \\
\hline Mean(SD) & $61.4(7.93)$ & 63.6(6.39) & $62.2(7.45)$ & $48.4(6.51)$ & $63.7(5.92)$ & $62.2(7.45)$ & $62.3(7.53)$ & $61.9(7.12)$ & $62.2(7.45)$ \\
\hline $\begin{array}{l}\text { Median [Min, } \\
\text { Max] }\end{array}$ & $\begin{array}{c}62.0 \\
{[38.0,78.0]}\end{array}$ & $\begin{array}{c}64.0 \\
{[46.0,77.0]}\end{array}$ & $\begin{array}{c}63.0 \\
{[38.0,78.0]}\end{array}$ & $\begin{array}{c}49.0 \\
{[38.0,78.0]}\end{array}$ & $\begin{array}{c}64.0 \\
{[51.0,77.0]}\end{array}$ & $\begin{array}{c}63.0 \\
{[38.0,78.0]}\end{array}$ & $\begin{array}{c}63.0 \\
{[38.0,78.0]}\end{array}$ & $\begin{array}{c}62.0 \\
{[39.0,75.0]}\end{array}$ & $63.0[38.0,78.0]$ \\
\hline \multicolumn{10}{|c|}{ Consultant } \\
\hline Consultant 1 & $18(8.7 \%)$ & $18(14.5 \%)$ & $36(10.9 \%)$ & $1(3.2 \%)$ & $35(11.7 \%)$ & $36(10.9 \%)$ & $34(12.2 \%)$ & $2(3.8 \%)$ & $36(10.9 \%)$ \\
\hline Consultant 2 & $42(20.3 \%)$ & $12(9.7 \%)$ & $54(16.3 \%)$ & $6(19.4 \%)$ & $48(16.0 \%)$ & $54(16.3 \%)$ & $50(18.0 \%)$ & $4(7.5 \%)$ & $54(16.3 \%)$ \\
\hline Consultant 3 & $13(6.3 \%)$ & $10(8.1 \%)$ & $23(6.9 \%)$ & $0(0 \%)$ & $23(7.7 \%)$ & $23(6.9 \%)$ & $20(7.2 \%)$ & $3(5.7 \%)$ & $23(6.9 \%)$ \\
\hline Consultant 4 & $78(37.7 \%)$ & $55(44.4 \%)$ & $133(40.2 \%)$ & $18(58.1 \%)$ & $115(38.3 \%)$ & $133(40.2 \%)$ & $107(38.5 \%)$ & $26(49.1 \%)$ & $133(40.2 \%)$ \\
\hline Consultant 5 & $56(27.1 \%)$ & $29(23.4 \%)$ & $85(25.7 \%)$ & $6(19.4 \%)$ & $79(26.3 \%)$ & $85(25.7 \%)$ & $67(24.1 \%)$ & $18(34.0 \%)$ & $85(25.7 \%)$ \\
\hline \multicolumn{10}{|c|}{ Pre-Op PSA } \\
\hline Mean(SD) & $10.6(8.64)$ & $9.83(7.37)$ & $10.2(8.05)$ & $8.82(8.22)$ & $10.3(7.83)$ & $9.56(8.02)$ & $9.62(7.73)$ & $12.7(8.14)$ & $11.2(7.94)$ \\
\hline Median & $8.00[1.80-68.0]$ & $\begin{array}{c}8.00[1.20- \\
64.5]\end{array}$ & $\begin{array}{c}8.00[1.20- \\
68.0]\end{array}$ & $\begin{array}{c}6.00[2.07- \\
31.6]\end{array}$ & $\begin{array}{c}8.24[1.20- \\
68.0]\end{array}$ & $\begin{array}{c}7.12[1.20- \\
68.0]\end{array}$ & $\begin{array}{c}7.87[1.20- \\
68.0]\end{array}$ & $\begin{array}{c}10.0[2.41- \\
33.0]\end{array}$ & $8.94[1.20-68.0]$ \\
\hline \multicolumn{10}{|c|}{ Gleason ISUP } \\
\hline 1 & $10(4.8 \%)$ & $9(7.3 \%)$ & $19(5.7 \%)$ & $1(3.2 \%)$ & $18(6.0 \%)$ & $19(5.7 \%)$ & $18(6.5 \%)$ & $1(1.9 \%)$ & $19(5.7 \%)$ \\
\hline $2+3$ & $170(82.1 \%)$ & $95(76.6 \%)$ & $265(80.1 \%)$ & $26(83.9 \%)$ & $239(79.7 \%)$ & $265(80.1 \%)$ & $227(81.7 \%)$ & $38(71.7 \%)$ & $265(80.1 \%)$ \\
\hline $4+5$ & $27(13.0 \%)$ & $20(16.1 \%)$ & $47(14.2 \%)$ & $4(12.9 \%)$ & $43(14.3 \%)$ & $47(14.2 \%)$ & $33(11.9 \%)$ & $14(26.4 \%)$ & $47(14.2 \%)$ \\
\hline \multicolumn{10}{|c|}{ T stage groups } \\
\hline $0-1$ & $143(69.1 \%)$ & $83(66.9 \%)$ & $226(68.3 \%)$ & $24(77.4 \%)$ & $202(67.3 \%)$ & $226(68.3 \%)$ & $196(70.5 \%)$ & $30(56.6 \%)$ & $226(68.3 \%)$ \\
\hline $2,3,4$ & $64(30.9 \%)$ & $41(33.1 \%)$ & $105(31.7 \%)$ & $7(22.6 \%)$ & $98(32.7 \%)$ & $105(31.7 \%)$ & $82(29.5 \%)$ & $23(43.4 \%)$ & $105(31.7 \%)$ \\
\hline \multicolumn{10}{|c|}{ Seminal vesicles invasion } \\
\hline 0 & $195(94.2 \%)$ & $115(92.7 \%)$ & $310(93.7 \%)$ & $29(93.5 \%)$ & $281(93.7 \%)$ & $310(93.7 \%)$ & $263(94.6 \%)$ & $47(88.7 \%)$ & $310(93.7 \%)$ \\
\hline 1 & $12(5.8 \%)$ & $9(7.3 \%)$ & $21(6.3 \%)$ & $2(6.5 \%)$ & $19(6.3 \%)$ & $21(6.3 \%)$ & $15(5.4 \%)$ & $6(11.3 \%)$ & $21(6.3 \%)$ \\
\hline \multicolumn{10}{|c|}{ Positive nodes } \\
\hline None & 191(92.3\%) & 113(91.1\%) & $304(91.8 \%)$ & 29(93.5\%) & $275(91.7 \%)$ & $304(91.8 \%)$ & $262(94.2 \%)$ & $42(79.2 \%)$ & $304(91.8 \%)$ \\
\hline Benign & $10(4.8 \%)$ & $9(7.3 \%)$ & 19(5.7\%) & $2(6.5 \%)$ & $17(5.7 \%)$ & $19(5.7 \%)$ & $12(4.3 \%)$ & $7(13.2 \%)$ & $19(5.7 \%)$ \\
\hline $\begin{array}{l}\text { Micrometastasis- } \\
\text { Metastasis }\end{array}$ & $6(2.9 \%)$ & $2(1.6 \%)$ & $8(2.4 \%)$ & $0(0 \%)$ & $8(2.7 \%)$ & $8(2.4 \%)$ & $4(1.4 \%)$ & $4(7.5 \%)$ & $8(2.4 \%)$ \\
\hline \multicolumn{10}{|c|}{ Margins } \\
\hline Negative & $168(81.2 \%)$ & $99(79.8 \%)$ & $267(80.1 \%)$ & $26(83.9 \%)$ & $241(80.3 \%)$ & $267(80.1 \%)$ & $234(84.2 \%)$ & $33(62.3 \%)$ & $267(80.1 \%)$ \\
\hline$<=3 \mathrm{~mm}$ & $26(12.6 \%)$ & $20(16.1 \%)$ & $46(13.9 \%)$ & $4(12.9 \%)$ & $42(14.0 \%)$ & $46(13.9 \%)$ & $28(10.1 \%)$ & $18(34.0 \%)$ & $46(13.9 \%)$ \\
\hline$>3 \mathrm{~mm}$ & $13(6.3 \%)$ & $5(4.0 \%)$ & $18(5.44 \%)$ & $1(3.2 \%)$ & $17(5.7 \%)$ & $180(5.44 \%)$ & $16(5.8 \%)$ & $2(3.8 \%)$ & $18(5.44 \%)$ \\
\hline \multicolumn{10}{|c|}{ Nerve Sparing } \\
\hline None & 40(19.3\%) & $30(24.2 \%)$ & 70(21.1\%) & $4(12.9 \%)$ & $66(22.0 \%)$ & $70(21.1 \%)$ & 49(17.6\%) & 21(39.6\%) & 70(21.1\%) \\
\hline Unilateral & $72(34.8 \%)$ & $37(29.8 \%)$ & $109(32.9 \%)$ & $7(22.6 \%)$ & $102(34.0 \%)$ & $109(32.9 \%)$ & $94(33.8 \%)$ & $15(28.3 \%)$ & $109(32.9 \%)$ \\
\hline Bilateral & $95(45.9 \%)$ & $57(46.0 \%)$ & $152(45.9 \%)$ & $20(64.5 \%)$ & $132(44.0 \%)$ & $152(45.9 \%)$ & $135(48.6 \%)$ & $17(32.1 \%)$ & $152(45.9 \%)$ \\
\hline
\end{tabular}

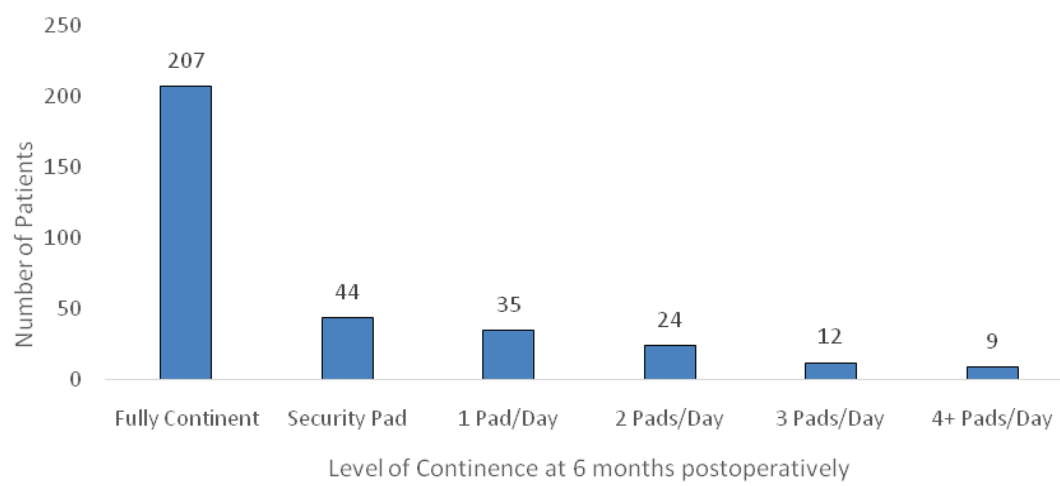

Figure 1: A graph showing the frequency of continence levels 6 months postoperatively. 
Table 2: Showing the multiple logistic regression analyses between the perioperative variables analysed comparative to the postoperative PSA levels at 6 weeks.(Ref=reference group, $\mathrm{OR}=$ odds ratio).

\begin{tabular}{|c|c|c|c|c|}
\hline & \multicolumn{4}{|c|}{ Post-Operative PSA } \\
\hline & OR & $2,5 \% \mathrm{Cl}$ & $97,5 \% \mathrm{Cl}$ & P-value \\
\hline Age & 1.00 & 0.96 & 1.04 & 0.86 \\
\hline \multicolumn{5}{|c|}{ Consultant } \\
\hline Consultant 1 & Ref & Ref & Ref & Ref \\
\hline Consultant 2 & 0.96 & 0.17 & 7.46 & 0.97 \\
\hline Consultant 3 & 2.68 & 0.41 & 21.85 & 0.31 \\
\hline Consultant 4 & 3.52 & 0.94 & 22.90 & 0.10 \\
\hline Consultant 5 & 3.33 & 0.84 & 22.31 & 0.13 \\
\hline Pre-Op PSA & 1.05 & 1.01 & 1.09 & $0.01 *$ \\
\hline \multicolumn{5}{|c|}{ Gleason ISUP } \\
\hline 1 & Ref & Ref & Ref & Ref \\
\hline $2+3$ & 3.21 & 0.62 & 58.91 & 0.27 \\
\hline $4+5$ & 6.10 & 1.02 & 117.51 & 0.10 \\
\hline \multicolumn{5}{|c|}{ T stage groups } \\
\hline $0-1$ & Ref & Ref & Ref & Ref \\
\hline $2,3,4$ & 1.17 & 0.57 & 2.34 & 0.66 \\
\hline \multicolumn{5}{|c|}{ Seminal vesicles invasion } \\
\hline 0 & Ref & Ref & Ref & Ref \\
\hline 1 & 1.20 & 0.34 & 3.79 & 0.76 \\
\hline \multicolumn{5}{|c|}{ Positive nodes } \\
\hline None & Ref & Ref & Ref & Ref \\
\hline Benign & 2.24 & 0.68 & 7.04 & 0.17 \\
\hline $\begin{array}{l}\text { Micrometastasis- } \\
\text { Metastasis }\end{array}$ & 4.74 & 0.96 & 23.99 & $0.05^{*}$ \\
\hline \multicolumn{5}{|c|}{ Margins } \\
\hline Negative & Ref & Ref & Ref & Ref \\
\hline$<=3 \mathrm{~mm}$ & 4.7 & 1.87 & 11.81 & $0.00 *$ \\
\hline$>3 \mathrm{~mm}$ & 1.46 & 0.27 & 8.05 & 0.66 \\
\hline \multicolumn{5}{|c|}{ Nerve Sparing } \\
\hline None & Ref & Ref & Ref & Ref \\
\hline Unilateral & 0.37 & 0.15 & 0.91 & $0.03 *$ \\
\hline Bilateral & 0.3 & 0.12 & 0.71 & $0.01^{*}$ \\
\hline
\end{tabular}

increasing age to be significantly associated to increased urinary incontinence $(\mathrm{OR}=1.04, \mathrm{p}=0.02)$ and we also found one consultant out of the five to be significantly associated to reduced incontinence outcomes $(\mathrm{OR}=0.33, \mathrm{p}=0.02)$ (Table 3$)$.

Erectile Dysfunction (ED) at 6 months: At 6 months patients' responses were categorized into the following outcomes: no ED; minimal or improving spontaneous erections; erections managed well with oral agents and/or a pump device; trial period with the same or another oral agent; erections not managed with oral agents and pump, hence trial injections; erections managed well with injections; erections not managed well with oral agents, pump and injections; and finally, certain patients did not wish to pursue treatment for ED. At 6 months $35.2 \%$ (116/331) patients were found to have no ED, minimal or improving spontaneous erections or were managing erections well with oral agents and pump (Figure 2).

On multiple logistic regression analysis perioperative variables regarding Operating Consultants and the presence of metastatic nodes were removed to avoid redundancies. From the analysis we found increasing age to be significantly associated with in increased levels of
Table 3: Showing the multiple logistic regression analyses between the perioperative variables analysed comparative to the postoperative urinary continence outcomes at 6 months. (Ref=reference group, OR=odds ratio).

\begin{tabular}{|c|c|c|c|c|}
\hline & \multicolumn{4}{|c|}{ Urinary Incontinence } \\
\hline & OR & $2,5 \% \mathrm{Cl}$ & $97,5 \% \mathrm{Cl}$ & P-value \\
\hline Age & 1.04 & 1.01 & 1.08 & $0.02 *$ \\
\hline \multicolumn{5}{|c|}{ Consultant } \\
\hline Consultant 1 & Ref & Ref & Ref & Ref \\
\hline Consultant 2 & 0.33 & 0.13 & 0.82 & $0.02 *$ \\
\hline Consultant 3 & 0.85 & 0.29 & 2.46 & 0.77 \\
\hline Consultant 4 & 0.83 & 0.39 & 1.80 & 0.64 \\
\hline Consultant 5 & 0.53 & 0.23 & 1.23 & 0.14 \\
\hline Pre-Op PSA & 1.02 & 0.99 & 1.05 & 0.19 \\
\hline \multicolumn{5}{|c|}{ Gleason ISUP } \\
\hline 1 & Ref & Ref & Ref & Ref \\
\hline $2+3$ & 0.64 & 0.24 & 1.70 & 0.36 \\
\hline $4+5$ & 0.81 & 0.26 & 2.55 & 0.72 \\
\hline \multicolumn{5}{|c|}{ T stage groups } \\
\hline $0-1$ & Ref & Ref & Ref & Ref \\
\hline $2,3,4$ & 0.98 & 0.57 & 1.68 & 0.94 \\
\hline \multicolumn{5}{|c|}{ Seminal vesicles invasion } \\
\hline 0 & Ref & Ref & Ref & Ref \\
\hline 1 & 1.11 & 0.39 & 3.16 & 0.84 \\
\hline \multicolumn{5}{|c|}{ Positive nodes } \\
\hline None & Ref & Ref & Ref & Ref \\
\hline Benign & 1.93 & 0.65 & 5.73 & 0.23 \\
\hline $\begin{array}{l}\text { Micrometastasis- } \\
\text { Metastasis }\end{array}$ & 0.55 & 0.08 & 2.65 & 0.49 \\
\hline \multicolumn{5}{|c|}{ Margins } \\
\hline Negative & Ref & Ref & Ref & Ref \\
\hline$<=3 \mathrm{~mm}$ & 1.36 & 0.64 & 2.9 & 0.43 \\
\hline$>3 \mathrm{~mm}$ & 0.71 & 0.21 & 2.38 & 0.58 \\
\hline \multicolumn{5}{|c|}{ Nerve Sparing } \\
\hline None & Ref & Ref & Ref & Ref \\
\hline Unilateral & 0.75 & 0.37 & 1.51 & 0.42 \\
\hline Bilateral & 0.96 & 0.49 & 1.89 & 0.9 \\
\hline
\end{tabular}

erectile dysfunction postoperatively at 6 months $(\mathrm{OR}=1.78, \mathrm{p}=0.00)$ (Table 4).

\section{Discussion}

This study provides interesting data relating to significant associations amongst perioperative variables and postoperative outcomes following RARP.

\section{PSA levels}

PSA levels after RARP are a significant marker in indication of operative success and oncological prognosis, the aim being to achieve undetectable levels of PSA $[10,11]$. Increased PSA prior to RARP would indicate a poorer general prognosis with a more advanced tumour staging and invasion of the cancer thus increasing the potential 
Table 4: Showing the multiple logistic regression analyses between the perioperative variables analysed comparative to the postoperative erectile function outcomes at 6 months.(ref=reference group, OR=odds ratio).

\begin{tabular}{|c|c|c|c|c|}
\hline & \multicolumn{4}{|c|}{ Erectile Dysfunction } \\
\hline & OR & $2,5 \% \mathrm{Cl}$ & $97,5 \% \mathrm{Cl}$ & P-value \\
\hline Age & 1.78 & 1.49 & 2.28 & $0.00 *$ \\
\hline Pre-Op PSA & 0.99 & 0.84 & 1.18 & 0.95 \\
\hline \multicolumn{5}{|c|}{ Gleason ISUP } \\
\hline 1 & Ref & Ref & Ref & Ref \\
\hline $2+3$ & 0.36 & 0.01 & 4.24 & 0.45 \\
\hline $4+5$ & 0.18 & 0.00 & 4.63 & 0.32 \\
\hline \multicolumn{5}{|c|}{ T stage groups } \\
\hline $0-1$ & Ref & Ref & Ref & Ref \\
\hline $2,3,4$ & 1.38 & 0.27 & 8.23 & 0.70 \\
\hline \multicolumn{5}{|c|}{ Seminal vesicles invasion } \\
\hline 0 & Ref & Ref & Ref & Ref \\
\hline 1 & 0.15 & 0.00 & 5.12 & 0.35 \\
\hline \multicolumn{5}{|c|}{ Margins } \\
\hline Negative & Ref & Ref & Ref & Ref \\
\hline$<=3 \mathrm{~mm}$ & 3.08 & 0.03 & 291.76 & 0.63 \\
\hline$>3 \mathrm{~mm}$ & 0.93 & 0.03 & 26.63 & 0.96 \\
\hline \multicolumn{5}{|c|}{ Nerve Sparing } \\
\hline None & Ref & Ref & Ref & Ref \\
\hline Unilateral & 0.5 & 0.04 & 6.92 & 0.61 \\
\hline Bilateral & 0.64 & 0.06 & 7.07 & 0.72 \\
\hline
\end{tabular}

of metastases and remnants of the cancer post resection. Our data supports the preoperative PSA levels determining post op levels as has been reported in previous literature $[12,13]$. We have also identified metastatic lymph node involvement compared to benign were also significantly associated to increased postoperative PSA levels further substantiating the literature $(\mathrm{OR}=4.74, \mathrm{p}=0.05)$.

Interestingly, multiple logistic regression analysis found unilateral and more so bilateral nerve sparing to be significantly associated to reduce PSA levels postoperatively at 6 weeks with odds ratios 0.37 and 0.30 respectively. This most likely is due to the reduced tumour stage of such patients as nerve sparing is only offered to non-metastasised compared to non-nerve sparing RARPs. The fine balance between the benefits of nerve sparing and the detriments of positive surgical margins as highlighted within this study should be further explored.

Positive margins less than $3 \mathrm{~mm}$ in length with an odds ratio of $4.70(1.87-11.8195 \% \mathrm{CI})$ to be significantly associated to increased postoperative PSA levels at 6 weeks, compared to negative surgical margins. This may have also been true for positive margins greater than $3 \mathrm{~mm}$ in length, however with only 18 such cases identified a significant association could not be stated, a larger study would be required to confirm. Nevertheless, if there are positive margins it is more likely that there is a localised spread of micrometastasis into the neighbouring structures, thus suggesting a higher PSA.
From the results achieved from this study the overall positive surgical margin rate was $19 \%$ compared to the national average of $31 \%$. Once separated by tumour staging we found T2 $(n=228)$ positive margin rates to be only $8 \%$ compared to the national average of $13 \%$, whereas T3a $(n=58)$ positive surgical margin rates were found to be significantly higher at $44.6 \%$ when the national average would aim for approximately $30 \%$ [7]. Following departmental consultant discussion, we believe the explanation were to aim to nerve spare and hence narrower excisions of T3a tumours. Regardless, we hope to further develop and implement quality assurance programmes as described by Cathcart P, et al. in 2015, whereby the use of monthly image-based surgical planning peer reviewed sessions will help us to understand how this discrepancy arose and provide solution on how to improve such outcomes in future [14].

\section{Urinary continence}

From the multiple logistic regression analysis, we found increasing age and the possibility of the operating consultant to significantly affect postoperative continence outcomes. The impact of increasing age has already been well documented in previous literature due to decline in nerve function/muscle strength alongside increasing comorbidities, such as heart conditions, arthritis, and diabetes with increasing age $[15,16]$.

This study is one of the first to identify a possible affect the operating consultant can have on postoperative continence outcomes. In our cohort five experienced consultant urologists performed the operations, however one of the consultants was associated to significantly improved continence outcomes postoperatively. Similar to Pick et al we cannot confirm a learning curve effect to be the primary explanation for this finding [16]. This is because all surgeons were highly experienced, operating independently as consultants for an excess of 10 years each. Therefore, if the learning curve cannot be the sole explanation for our significant difference reported, other factors should be considered.

The surgeon with improved continence results had a varying surgical technique; aiming for maximal apical dissection and urethral length preservation; ensuring no endopelvic fascia dissection; meticulous bladder neck reconstruction; cold cutting of the dorsal venous complex and the use of larger urethral suture bites. He believes these techniques may have affected the continence outcomes and we therefore hope to explore the intricacies of the operative technique in future studies. Another potential variable factor may lie with the experience of the bedside operating assistant. Although the bedside assistant operates under close supervision of the consultant at the console, a learning curve also exists for the assistant, in addition to variations in the surgical technique which should be explored.

However, as the data was collected retrospectively, we cannot be completely certain if this association identified was limited by unintentional biases. This is firstly due to each consultant urologist operating upon a different volume of cases, ranging with the highest performing 133 RARPs and the lowest only 23. In addition, confounding variables such as age as identified in this study, alongside tumour staging and preoperative continence levels which could significantly affect postoperative continence outcomes were not controlled and thus could also affect this association identified.

\section{Erectile dysfunction}

Erectile function is a very important functional outcome for patients, allowing for improved recovery and quality of life postoperatively. Our study identified increasing age which is well 


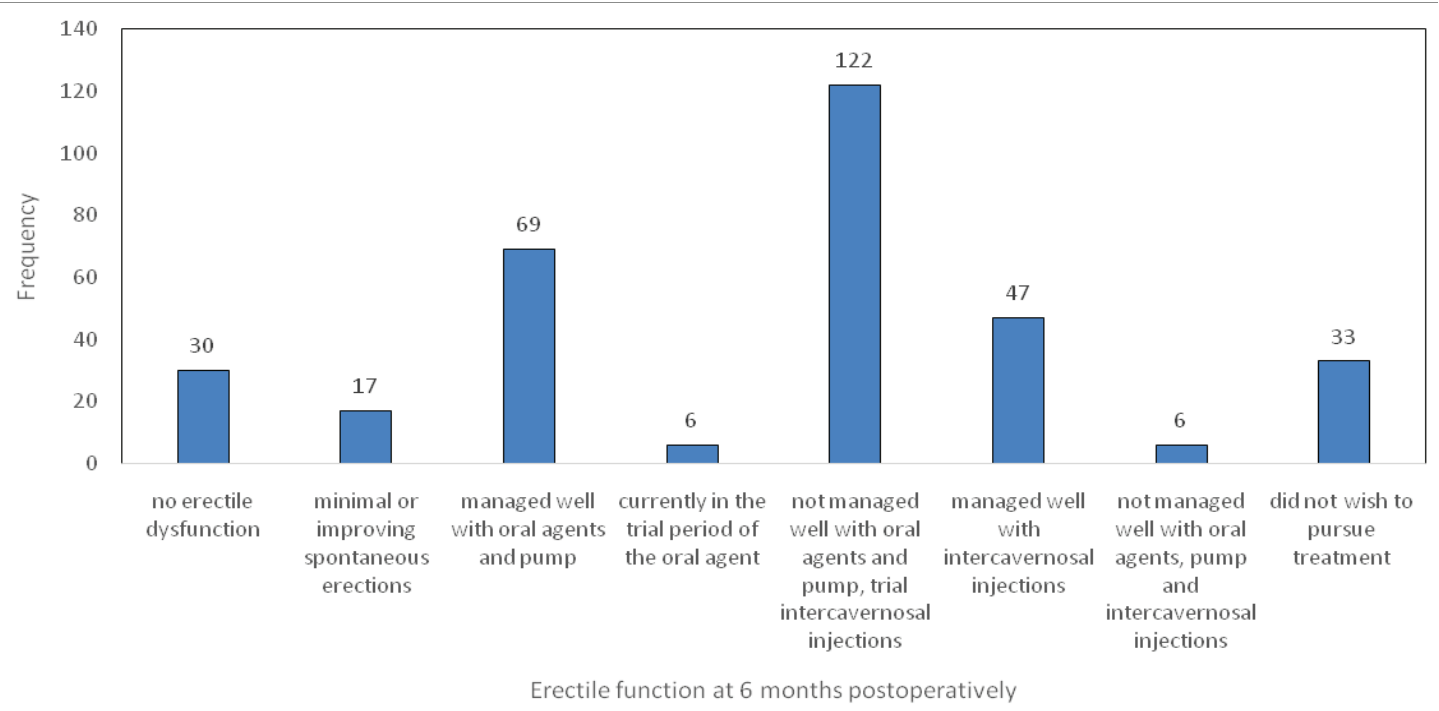

Figure 2: A graph showing the frequency of erectile dysfunction outcomes 6 months postoperatively.

documented in the literature due to many factors such as declining nerve function, increasing co-morbidities, reduced testosterone levels and psychological distress [17]. The negative correlations are also useful to note whereby our results found perioperative variables such as preoperative PSA scores, presence of histopathological tumor invasion and increased tumour size and staging showed no statistically significant relationship with erectile function postoperative outcomes. We hope our results can help support the literature further and thus manage patient outcomes, especially amongst a more elderly population undergoing the RARP procedure $[5,18]$.

\section{Limitations of this study}

Due to the retrospective nature of the study, we had a limited sample size, increasing this would increase our reliability. A formal ED assessment tool wasn't used or a comment on the patient's comorbidities. In the future we aim to implement this to aid patient counselling and management of expectations, whilst also providing additional explanation of these study findings. Also, for the outcomes concerning erectile dysfunction $10 \%$ of patients did not wish to pursue treatment which may have also altered our results as well.

\section{Conclusion}

Postoperative PSA levels were significantly increased in patients associated with high preoperative PSA levels, metastatic lymph nodes and those with positive surgical margins less than $3 \mathrm{~mm}$ in length whilst significantly decreased amongst patients undergoing unilateral and bilateral nerve sparing RARP's. Increasing age and the effect of the operating consultant were also found to significantly affect continence outcomes postoperatively. We believe surgical technique impacts continence as detailed above and will look to explore this further. Overall this study will help predict postoperative functional outcomes in patients undergoing RARPs based on perioperative data allowing the urologist to be more informed of their patient's functional prognosis, aid counselling of patient expectations, in addition to oncological outcomes.

\section{Acknowledgment and Disclosure Statement}

No further acknowledgements or competing financial interests exist. Ethical approval was not required as this study focused on retrospective anonymised data analysis. This study has been registered with Research Registry under registration ID 5785.

\section{References}

1. Binder J, Kramer W (2001) Robotically-assisted laparoscopic radical prostatectomy. BJU Int 87: 408-410.

2. Heidenreich A, Varga Z, Von Knobloch R (2002) Extended pelvic lymphadenectomy in patients undergoing radical prostatectomy: high incidence of lymph node metastasis. J Urol 167: 1681-1686.

3. Pasticier G, Rietbergen JB, Guillonneau B, Fromont G, Menon M, et al. (2001) Robotically assisted laparoscopic radical prostatectomy: feasibility study in men. Eur Urol 40: 70-74.

4. Rassweiler J, Frede T, Seemann O, Stock C, Sentker L (2001) Telesurgical laparoscopic radical prostatectomy. Initial experience. Eur Urol 40: 75-83.

5. Ficarra V, Novara G, Ahlering TE, Costello A, Eastham JA, et al. (2012) Systematic review and meta-analysis of studies reporting potency rates after robot-assisted radical prostatectomy. Eur Urol 62: 418430.

6. Montorsi F, Wilson TG, Rosen RC, Ahlering TE, Artibani W, et al. (2012) Best practices in robot-assisted radical prostatectomy: recommendations of the Pasadena Consensus Panel. Eur Urol 62: 368-381.

7. London Cancer Alliance L. LCA Urological Cancer Clinical Guidelines 2014.

8. Agha R, Abdall-Razak A, Crossley E, Dowlut N, losifidis C, et al. (2019) STROCSS 2019 Guideline: Strengthening the reporting of cohort studies in surgery. Int J Sur 72: 156-165.

9. Epstein JI, Zelefsky MJ, Sjoberg DD, Nelson JB, Egevad L, et al. (2016) A contemporary prostate cancer grading system: a validated alternative to the gleason score. Eur Urol 69: 428-435.

10. Suardi N, Ficarra V, Willemsen P, De Wil P, Gallina A, et al. (2012) Long-term biochemical recurrence rates after robot-assisted radical prostatectomy: analysis of a single-center series of patients with a minimum follow-up of 5 years. Urology 79: 133-138. 
11. Sooriakumaran $\mathrm{P}$, Haendler L, Nyberg T, Gronberg H, Nilsson A, et al. (2012) Biochemical recurrence after robot-assisted radical prostatectomy in a European single-centre cohort with a minimum follow-up time of 5 years. Eur Urol 62: 768-774.

12. D'Amico AV, Whittington R, Malkowicz SB, Fondurulia J, Chen $\mathrm{MH}$, et al. (1998) The combination of preoperative prostate specific antigen and postoperative pathological findings to predict prostate specific antigen outcome in clinically localized prostate cancer. J Urol 160: 2096-2101.

13. Oesterling JE, Chan DW, Epstein JI, Kimball AW, Jr Bruzek DJ, et al. (1988) Prostate specific antigen in the preoperative and postoperative evaluation of localized prostatic cancer treated with radical prostatectomy. J Urol 139: 766-772.

14. Cathcart P, Sridhara A, Ramachandran N, Briggs T, Nathan S, et al. (2015) Achieving quality assurance of prostate cancer surgery during reorganisation of cancer services. Eur Urol 68: 22-29.
15. Pick DL, Osann K, Skarecky D, Narula N, Finley DS, et al. (2011) The impact of cavernosal nerve preservation on continence after robotic radical prostatectomy. BJU Int 108: 1492-1496.

16. Lee DJ, Cheetham P, Badani KK (2010) Predictors of early urinary continence after robotic prostatectomy. Can J Urol 17: 5200-5205.

17. Woo SH, Kang DI, Ha YS, Salmasi AH, Kim JH, et al. (2014) Comprehensive analysis of sexual function outcome in prostate cancer patients after robot-assisted radical prostatectomy. J Endourol 28: 172-177.

18. Orvieto MA, Coelho RF, Chauhan S, Mathe M, Palmer K, et al. (2010) Erectile dysfunction after robot-assisted radical prostatectomy. Expert Rev Anticancer Ther 10: 747-754. 